\title{
Some Dynamic Graphics for Spatial Data (with Multiple Attributes) in a GIS
}

\author{
Dianne Cook $^{1}$, Noel Cressie ${ }^{1}$, James Majure ${ }^{2}$, Jürgen Symanzik ${ }^{1}$ \\ 1 Department of Statistics, Iowa State University, Ames, IA 50011, USA \\ ${ }^{2}$ GIS Support and Research Facility, Iowa State University \\ dicook@iastate.edu
}

\begin{abstract}
This paper discusses some multivariate exploratory spatial data analysis tools for detecting spatial dependence. The ideas explored are related to canonical correlation analysis and the graphical tools are related to the dynamic method called the grand tour. The work is implemented with a link between a Geographic Information System, ARC/INFO ${ }^{\mathrm{TM}}$, and software for exploring multivariate data, XGobi.
\end{abstract}

Keywords. canonical correlations, correlation tour, exploratory spatial data analysis, spatial dependence, spatial statistics

\section{Introduction}

\subsection{Terminology and Notation for Spatial Data}

Spatial methods involve acknowledgement of the spatial location $s$ of the attribute $\boldsymbol{Z}(\boldsymbol{s}) ; \boldsymbol{s} \in D$, where $D$ is a subset of $d$-dimensional space. In what follows we shall mainly be interested in a multivariate attribute:

$$
Z(s)=\left(Z_{1}(s), \ldots, Z_{p}(s)\right)^{\prime}
$$

When we investigate how average values of $\boldsymbol{Z}$ vary with $\boldsymbol{s}$, then we are considering the trend or large-scale variation in the attribute. One could think of such summaries as estimating

$$
\boldsymbol{\mu}(\boldsymbol{s})=\mathrm{E}[\boldsymbol{Z}(\boldsymbol{s})] ; \boldsymbol{s} \in D .
$$

Define the small-scale variation as $\delta(s) \equiv Z(s) \Leftrightarrow \boldsymbol{\mu}(s)$. Typically, some form of second-moment stationarity of $\delta(\boldsymbol{s})$ is assumed to allow the spatial dependence to be characterized and estimated. Therefore, in the spatial statistical model-building stage, it is important to have available methods for detecting regional variation in the attribute values, "pockets" of non-stationarity, and spurious data points (Cressie, 1984; Getis and Ord, 1992). Prediction based on the squared error loss function relies on the covariance properties of $\boldsymbol{Z}(\boldsymbol{s})$. In the multivariate setting we need to know $\operatorname{cov}\left(Z_{j}(s), Z_{j^{\prime}}\left(\boldsymbol{s}^{\prime}\right)\right), \forall j, j^{\prime}$ and $\forall \boldsymbol{s}, \boldsymbol{s}^{\prime}$; that is, we need to know $\operatorname{cov}\left(\boldsymbol{Z}(\boldsymbol{s}), \boldsymbol{Z}\left(\boldsymbol{s}^{\prime}\right)\right)=\mathrm{E}\left[\boldsymbol{\delta}(\boldsymbol{s}) \boldsymbol{\delta}\left(\boldsymbol{s}^{\prime}\right)^{\prime}\right] \equiv C\left(\boldsymbol{s}, \boldsymbol{s}^{\prime}\right)$, where $C(\cdot, \cdot)$ is a $p \times p$ covariance matrix. A stationarity assumption on $C$ leads to 


$$
\mathrm{E}\left[\boldsymbol{\delta}(\boldsymbol{s}+\boldsymbol{h}) \boldsymbol{\delta}(\boldsymbol{s})^{\prime}\right]=C(\boldsymbol{h}),
$$

a matrix function only of $\boldsymbol{h}$, the spatial lag. Thus, to characterize the spatial dependence in the small-scale variation we look at co-dependencies between $\boldsymbol{\delta}(\boldsymbol{s}+\boldsymbol{h})$ and $\boldsymbol{\delta}(\boldsymbol{s})$. This will be developed further in Section 2.1 and 2.2 . Section 2.3 discusses interactive and dynamic graphical methods to search for spatial dependence. A new component of our work is to combine the strengths of interactive and dynamic graphical methods with the spatial data base management and spatial functionalities of a Geographic Information System (GIS). A GIS is a computer hardware and software system designed to carry out a number of important tasks on spatially referenced data (see, for example, Maguire, 1991; Goodchild, 1991). This work is discussed in Section 3.

\subsection{Examples of Interactive and Dynamic Graphics Applied to Spatial Data}
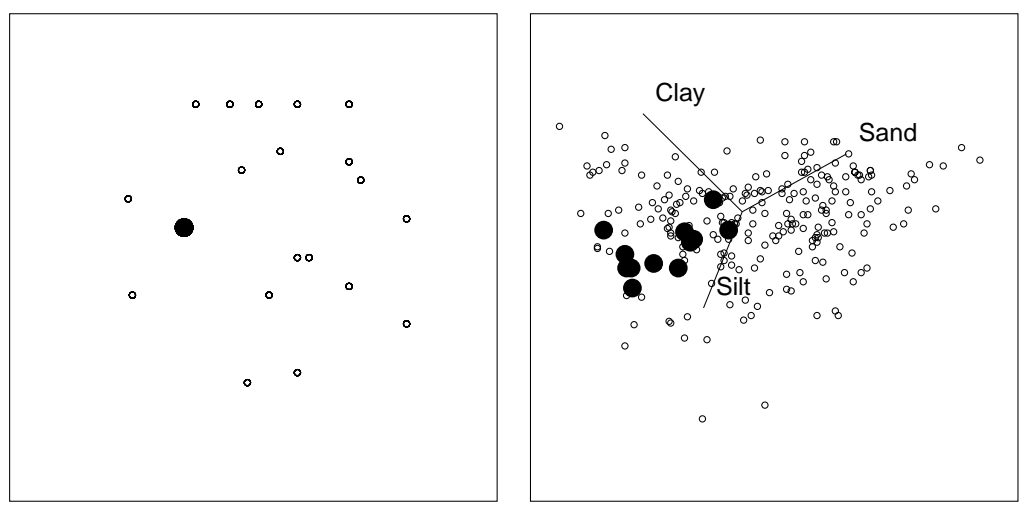

Figure 1: California soils data - linking the map view to the attribute display.

A natural approach to visualizing spatial data is to display the two groups of variables $(s, \boldsymbol{Z}(\boldsymbol{s}))$ using appropriate graphics in two separate windows. For example, consider the situation in Figure 1 where three measurements have been collected at many geographic sites; the collection sites are displayed as a 2-dimensional scatterplot of the geographic location (called a "map view" by Haslett et al., 1991), and the measurement data displayed as a 3-dimensional rotating or grand touring (Asimov, 1985; Buja and Asimov, 1986) point cloud. Because it is difficult to illustrate and describe in a static medium like the printed page, an important part of our research involves dynamic motion of some or all of the displays. The rotating and grand touring referred to earlier are two of a number of dynamic graphical methods that are extremely effective in illustrating structure in data, particularly multivariate data. 
Each plot in Figure 1 is linked dynamically so that highlighting (or brushing or identifying) in one plot simultaneously causes equivalent highlighting in the other plots. Plot (a) shows the geographic data corresponding to 20 locations in an undisclosed area of California and plot (b) is a 2-dimensional snapshot of a 3-dimensional point cloud of the variables sand, silt, and clay. (The data can be found in Andrews and Herzberg, 1985, set 16. Measurements are also taken at 12 different depths at each site.) At the site highlighted $(\bullet)$ the soil can be (and actually is) classified as silty clay, an almost equal mixture of silt and clay particle sizes with very little sand. ${ }^{1}$

Finding clusters in the image space has been fairly successfully done by using multivariate methods for clustering on the attribute data and linking this to the spatial variable plot. McDonald and Willis (1987) (discussed also in Buja et al., 1991) use the grand tour on 4 Landsat MSS spectral bands to identify forest, grasslands, muddy water of the Rio Solimões, and the dark water of the Rio Negro in an image taken of an area near Manaus, Brazil. They show that a simple linear mixing model for the confluence of the two rivers is sufficient.
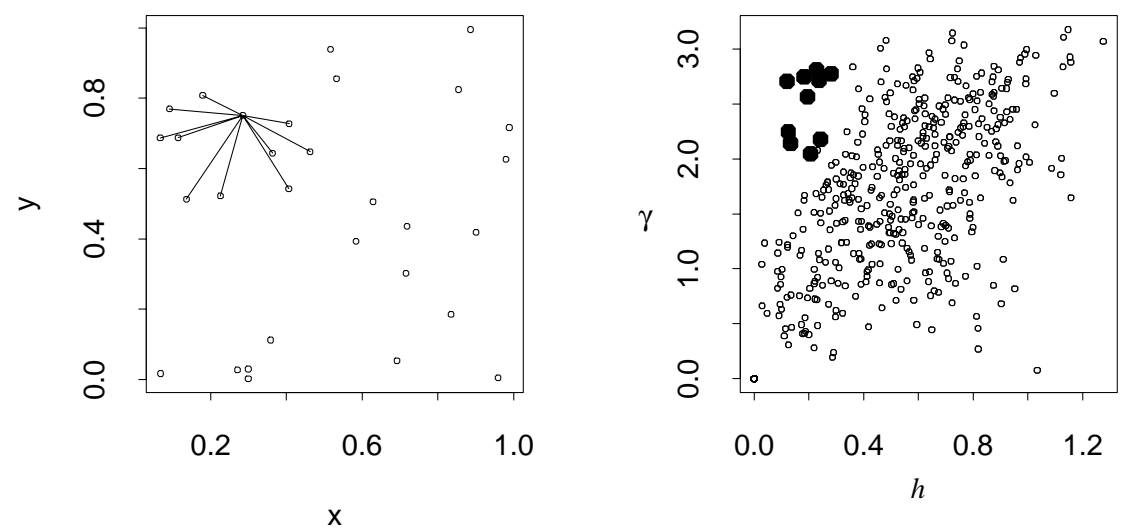

Figure 2: Using linking between the map view and variogram cloud to detect an outlying point. (The data is simulated purely to illustrate the method.)

Graphical methods developed for spatial data are enhanced by incorporation into the linked window approach. When there is only one attribute variable, $Z(\boldsymbol{s})$, an isotropic variogram cloud (Chauvet, 1982; Cressie, 1984), $\gamma_{i j}=\left|Z\left(s_{i}\right) \Leftrightarrow Z\left(s_{j}\right)\right|^{1 / 2}$ versus $h_{i j}=\left\|s_{i} \Leftrightarrow s_{j}\right\|^{1 / 2}$ can be linked to the spatial variable plot. Brushing in the variogram cloud links to pairs of points

\footnotetext{
${ }^{1}$ An interesting aside to the data is that there are two reasonably large errors in the recording of the soil particle percentages. Because they are percentages, the points should lie in a 2-dimensional simplex in $\mathbb{R}^{3}$. During rotation, though, it is clear that 2 points do not lie in this region. Using identification and referring back to the data confirms that the (plot 4, depth 3) percentages sum to $109.1 \%$ and the (plot 3, depth 11) percentages sum to $96.3 \%$.
} 
connected by lines in the spatial variable plot. Interesting points (potential outliers or points defining the borders of spatial regions) that are spatially close but differ greatly in the attribute variable value are easily identified by this technique (Haslett et al., 1991; Bradley and Haslett, 1992). In Figure 2 , a spurious point is identified. The points $(\bullet)$ in the variogram cloud (plot (b)) that have large values of $\gamma_{i j}$ despite low spatial distance, $h_{i j}$, link back to the pairs of points in the map view (plot (a)) connected by lines. All the lines have one common end point indicating a location that has an attribute value differing substantially from its neighbours.

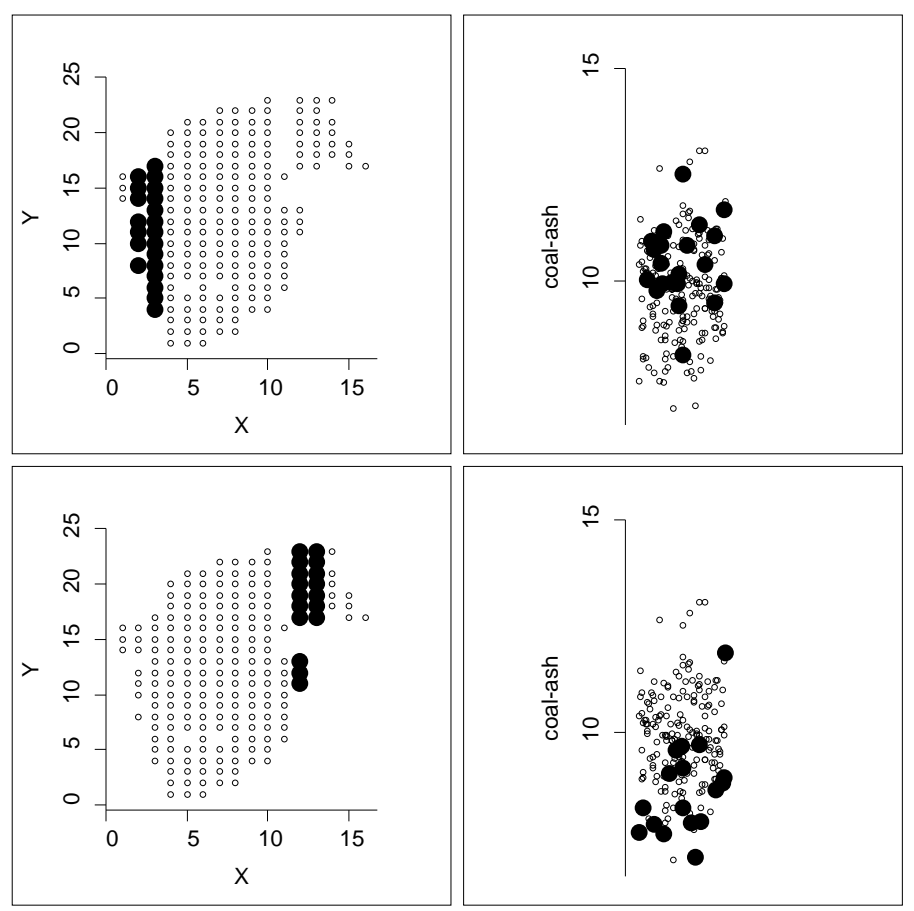

Figure 3: Detecting east-west trend in the coal-ash data (Cressie, 1993) by linked brushing.

In the coal ash example in Cressie (1993, Section 2.2), an east-west trend is detected by averaging attribute values, that is, taking means and also medians, over the north-south direction. Graphically (and interactively) this is equivalent to dragging a long narrow brush sideways and watching the change in distribution of highlighted points in the plot of the attribute variable (Figure 3). For higher dimensional $(\geq 3)$ attribute data, a crude approach is to divide the data into several colored groups by brushing regionally in the spatial plot and examining the relative positions of the colored groups during a grand tour. 


\section{Detecting Spatial Dependence}

\subsection{Analytical Tools}

The essence of most investigations into spatial dependence is to consider relationships between attributes and their lagged (neighbouring) versions. For this section, we shall assume that the trend component, pockets of nonstationarity, and spurious points have been removed from $Z(s)$.

For the moment, let us fix $\boldsymbol{h}$. We may vary $\boldsymbol{h}$ later if it is appropriate. Our approach is to concatenate $\boldsymbol{Z}(\boldsymbol{s}+\boldsymbol{h})$ with $\boldsymbol{Z}(\boldsymbol{s})$ and apply statistical methods to $\left(\boldsymbol{Z}(\boldsymbol{s}+\boldsymbol{h})^{\prime}, \boldsymbol{Z}(\boldsymbol{s})^{\prime}\right)^{\prime}$. For example, consider

$$
\begin{aligned}
\operatorname{var}\left(\begin{array}{c}
Z(\boldsymbol{s}+\boldsymbol{h}) \\
\boldsymbol{Z}(\boldsymbol{s})
\end{array}\right) & =\left[\begin{array}{cc}
\Sigma(\boldsymbol{s}) & C(\boldsymbol{s}+\boldsymbol{h}, \boldsymbol{s}) \\
C(\boldsymbol{s}, \boldsymbol{s}+\boldsymbol{h}) & \Sigma(\boldsymbol{s}+\boldsymbol{h})
\end{array}\right] \\
& =\left[\begin{array}{cc}
\Sigma & C(\boldsymbol{h}) \\
C(\Leftrightarrow \boldsymbol{h}) & \Sigma
\end{array}\right]
\end{aligned}
$$

under an assumption of stationarity of the covariance matrix. Henceforth, this stationarity is assumed. Notice that $C(\boldsymbol{h})$ is not necessarily symmetric, although it is square, and $C(\Leftrightarrow \boldsymbol{h})=C(\boldsymbol{h})^{\prime}$. When $\boldsymbol{h}=\mathbf{0}, C(\mathbf{0})=\Sigma$, which is symmetric.

Consider the singular value decomposition (SVD) of $\Sigma^{-1 / 2} C(\boldsymbol{h}) \Sigma^{-1 / 2}$, that is,

$$
\Sigma^{-1 / 2} C(\boldsymbol{h}) \Sigma^{-1 / 2}=U T V^{\prime}
$$

where $T=\operatorname{diag}\left(\tau_{1}, \ldots, \tau_{r}\right), \tau_{i}>0 \forall i, r$ is the rank of $C(\boldsymbol{h}), U$ is $p \times r, U^{\prime} U=$ $I, V^{\prime}$ is $r \times p, V^{\prime} V=I$. The $\left\{\tau_{i}^{2}\right\}$ are eigenvalues of $\Sigma^{-1 / 2} C(\boldsymbol{h})^{\prime} \Sigma^{-1} C(\boldsymbol{h}) \Sigma^{-1 / 2}$, or of $\Sigma^{-1 / 2} C(\boldsymbol{h}) \Sigma^{-1} C(\boldsymbol{h})^{\prime} \Sigma^{-1 / 2}$. The $r$ columns of $U$ are the eigenvectors of $\Sigma^{-1 / 2} C(\boldsymbol{h}) \Sigma^{-1} C(\boldsymbol{h})^{\prime} \Sigma^{-1 / 2}$ and the $r$ rows of $V^{\prime}$ are the eigenvectors of $\Sigma^{-1 / 2} C(\boldsymbol{h})^{\prime} \Sigma^{-1} C(\boldsymbol{h}) \Sigma^{-1 / 2}$. Then, $U^{\prime} \Sigma^{-1 / 2} C(\boldsymbol{h}) \Sigma^{-1 / 2} V=T=\operatorname{diag}\left(\tau_{1}, \ldots, \tau_{r}\right)$. Suppose we define $P=\Sigma^{-1 / 2} U$ and $Q=\Sigma^{-1 / 2} V$. Then:

$$
\begin{array}{rll}
U^{\prime} U=I & \Leftrightarrow & P^{\prime} \Sigma P=I \\
V^{\prime} V=I & \Leftrightarrow & Q^{\prime} \Sigma Q=I \\
U^{\prime} \Sigma^{-1 / 2} C(\boldsymbol{h}) \Sigma^{-1 / 2} V=T & \Leftrightarrow & P^{\prime} C(\boldsymbol{h}) Q=T=\operatorname{diag}\left(\tau_{1}, \ldots, \tau_{r}\right) .
\end{array}
$$

Now consider the problem of maximizing

$$
\operatorname{corr}\left(\boldsymbol{l}^{\prime} \boldsymbol{Z}(\boldsymbol{s}+\boldsymbol{h}), \boldsymbol{m}^{\prime} \boldsymbol{Z}(\boldsymbol{s})\right)
$$

with respect to $\boldsymbol{m}, \boldsymbol{l}$. Notice that the minimum/maximum autocorrelation factors (MAFs) of Switzer and Green (1984) are a special case with $\boldsymbol{l}=\boldsymbol{m}$ (Cressie and Helterbrand, 1994). Hence the problem above is more general and the correlation can achieve a higher maximum. We see that

$$
\operatorname{corr}\left(\boldsymbol{l}^{\prime} \boldsymbol{Z}(\boldsymbol{s}+\boldsymbol{h}), \boldsymbol{m}^{\prime} \boldsymbol{Z}(\boldsymbol{s})\right)=\frac{\boldsymbol{l}^{\prime} C(\boldsymbol{h}) \boldsymbol{m}}{\left\{\boldsymbol{l}^{\prime} \Sigma \boldsymbol{l} \cdot \boldsymbol{m}^{\prime} \Sigma \boldsymbol{m}\right\}^{1 / 2}}
$$


Under the conditions $\boldsymbol{l}^{\prime} \Sigma \boldsymbol{l}=\boldsymbol{m}^{\prime} \Sigma \boldsymbol{m}=1$, it is immediate that

$$
\operatorname{var}\left(\boldsymbol{l}^{\prime} \boldsymbol{Z}(\boldsymbol{s}+\boldsymbol{h}) \Leftrightarrow \boldsymbol{m}^{\prime} \boldsymbol{Z}(\boldsymbol{s})\right)=2\left\{1 \Leftrightarrow \operatorname{corr}\left(\boldsymbol{l}^{\prime} \boldsymbol{Z}(\boldsymbol{s}+\boldsymbol{h}), \boldsymbol{m}^{\prime} \boldsymbol{Z}(\boldsymbol{s})\right)\right\} .
$$

Hence maximizing the correlation is equivalent to minimizing the variability of (a linear combination of) $\boldsymbol{Z}(\boldsymbol{s})$ contrasted with (a different linear combination) of the neighbouring vector $\boldsymbol{Z}(\boldsymbol{s}+\boldsymbol{h})$.

From standard results in in canonical correlation analysis (e.g., Dillon and Goldstein, pp. 340-2), the problem is equivalent to solving the following canonical equations:

$$
\left(\Sigma^{-1} C(\boldsymbol{h}) \Sigma^{-1} C(\boldsymbol{h})^{\prime} \Leftrightarrow \lambda I\right) \boldsymbol{l}=\mathbf{0} \text { and }\left(\Sigma^{-1} C(\boldsymbol{h})^{\prime} \Sigma^{-1} C(\boldsymbol{h}) \Leftrightarrow \lambda I\right) \boldsymbol{m}=\mathbf{0} .
$$

The largest eigenvalue, $\lambda$, of the product matrix $\Sigma^{-1} C(\boldsymbol{h}) \Sigma^{-1} C(\boldsymbol{h})^{\prime}$ or of $\Sigma^{-1} C(\boldsymbol{h})^{\prime} \Sigma^{-1} C(\boldsymbol{h})$ is the squared canonical correlation coefficient. It can be shown that the associated eigenvectors satisfy

$$
\boldsymbol{l}=\Sigma^{-1} C(\boldsymbol{h}) \boldsymbol{m} / \sqrt{\lambda} \text { and } \boldsymbol{m}=\Sigma^{-1} C(\boldsymbol{h})^{\prime} \boldsymbol{l} / \sqrt{\lambda} .
$$

We normalize $\boldsymbol{l}$ and $\boldsymbol{m}$ so that $\boldsymbol{l}^{\prime} \Sigma \boldsymbol{l}=\boldsymbol{m}^{\prime} \Sigma \boldsymbol{m}=1$. Thus, the SVD analysis and the canonical correlation analysis are the same, where the canonical correlation coefficient $\sqrt{\lambda}=\tau_{1}, \boldsymbol{l}=\boldsymbol{p}_{1}$ (the first column of $P$ from the SVD), and $\boldsymbol{m}=\boldsymbol{q}_{1}$ (the first column of $Q$ from the SVD).

\subsection{Multivariate Spatial Models}

The use of canonical correlations and principal components to determine the presence of common factors is well known in multivariate analysis (Anderson, 1958, for example). There is also a considerable literature on canonical correlations for multivariate time series, where the presence of temporal dependence gives a new perspective to the approach (for example, Akaike, 1976; Box and Tiao, 1977; Cooper and Wood, 1982; Pena and Box, 1987; Aoki, 1987; Tiao and Tsay, 1989). There are two related features of this time series literature that make their generalization to spatial data unnatural. First, the notion of "past, present, and future" is important for the methods that use canonical correlations of past behaviour with present and future behaviour. Second, the models used are of the autoregressive-movingaverage type, which has no natural analogue in space (Cressie, 1993, Section 6.3). Wackernagel (1988) has proposed spatial common-factor models that do not require autoregressions or moving averages for their definitions. Both result in symmetric $C(\boldsymbol{h})$ and so are considered to be too specialized. We now consider a class of models that includes Wackernagel's, but, in general, allows a non-symmetric covariance matrix function $C(\boldsymbol{h})$.

The class of models considered are the shifted-lag models, defined as

$$
Z_{j}(\boldsymbol{s})=\sum_{i=1}^{k} a_{i j} W_{i}\left(\boldsymbol{s}+\boldsymbol{\Delta}_{i j}\right)+\varepsilon_{j}(\boldsymbol{s}), \quad j=1, \ldots, p,
$$


where $\left\{W_{1}(\cdot), \ldots, W_{k}(\cdot), \varepsilon_{1}(\cdot), \ldots, \varepsilon_{p}(\cdot)\right\}$ are pairwise independent (or at least uncorrelated). There are $p \times k$ lag vectors $\left\{\boldsymbol{\Delta}_{i j}: i=1, \ldots, p ; j=1, \ldots, k\right\}$ that are in general unknown parameters of the model. The unknown parameters $\left\{a_{i j}: i=1, \ldots, p ; j=1, \ldots, k\right\}$ are made identifiable by assuming, for example, that $\operatorname{var}\left(W_{i}(\boldsymbol{s})\right) \equiv 1$. Finally, $\varepsilon_{j}(\cdot)$ is a white-noise process with $v_{j}^{2} \equiv \operatorname{var}\left(\varepsilon_{j}(s)\right) ; j=1, \ldots, p$. For example, if $k=1$ and $p=2$, $Z_{1}(s)$ and $Z_{2}(s)$ derive their co-dependence from a common spatial process $W_{1}$. Without loss of generality, assume that $\boldsymbol{\Delta}_{11}=\mathbf{0}$ and $a_{11}=1$; then $Z_{1}(\boldsymbol{s})=W_{1}(\boldsymbol{s})+\varepsilon_{1}(\boldsymbol{s})$ and $Z_{2}(\boldsymbol{s})=a_{12} W_{1}\left(\boldsymbol{s}+\boldsymbol{\Delta}_{12}\right)+\varepsilon_{2}(\boldsymbol{s})$.

Assume further that each $W_{i}$ is second-order stationary. Then, for $j \neq j^{\prime}$, the $\left(j, j^{\prime}\right)$-th element of $C(\boldsymbol{h})$ is

$$
\operatorname{cov}\left(Z_{j}(\boldsymbol{s}+\boldsymbol{h}), Z_{j^{\prime}}(\boldsymbol{s})\right)=\sum_{i} a_{i j} a_{i j^{\prime}} C_{i}\left(\boldsymbol{h}+\boldsymbol{\Delta}_{i j} \Leftrightarrow \boldsymbol{\Delta}_{i j^{\prime}}\right),
$$

where $C_{i}(\boldsymbol{h}) \equiv \operatorname{cov}\left(W_{i}(\boldsymbol{s}+\boldsymbol{h}), W_{i}(\boldsymbol{s})\right) ; i=1, \cdots, k$. Also, the $j$-th diagonal element of $C(\boldsymbol{h})$ is

$$
\operatorname{var}\left(Z_{j}(\boldsymbol{s})\right)=\sum_{i} a_{i j}^{2}+v_{j}^{2} ; j=1, \ldots, p .
$$

Notice that $C(\boldsymbol{h}) \neq C(\Leftrightarrow \boldsymbol{h})$ unless $\boldsymbol{\Delta}_{i j}$ does not depend on $j$. When $\boldsymbol{\Delta}_{i j}$ is actually $\boldsymbol{\Delta}_{i}, C(\boldsymbol{h})$ is symmetric and the shifted-lag models reduce to those of Wackernagel (1988). Further, $\operatorname{cov}\left(Z_{j}(\boldsymbol{s}), W_{i}(\boldsymbol{s}+\boldsymbol{h})\right)=a_{i j} C_{i}\left(\boldsymbol{h} \Leftrightarrow \boldsymbol{\Delta}_{i j}\right)$. This function is symmetric about $\boldsymbol{h}=\boldsymbol{\Delta}_{i j}$. Thus, an indication of the value of $\boldsymbol{\Delta}_{i j}$ is obtained by shifting $C_{i}(\cdot)$ until it is symmetric.

However, $W_{i}(\cdot)$ is unknown. For the purpose of exploratory analysis, we propose to use a part of the spatial process that "looks like" $W_{i}(\cdot)$ in a number of important ways. We shall now establish that $\boldsymbol{p}_{i}^{\prime} \boldsymbol{Z}(\boldsymbol{s}+\boldsymbol{h}) \Leftrightarrow \boldsymbol{q}_{i}^{\prime} \boldsymbol{Z}(\boldsymbol{s})$ is a substitute for $W_{i}(\boldsymbol{s})$, where $\boldsymbol{p}_{i}=\Sigma^{-1 / 2} \boldsymbol{u}_{i}, \boldsymbol{q}_{i}=\Sigma^{-1 / 2} \boldsymbol{v}_{i},\left\{\boldsymbol{u}_{i}\right\}$ are the $r$ eigenvectors of $\Sigma^{-1 / 2} C(\boldsymbol{h}) \Sigma^{-1} C(\boldsymbol{h})^{\prime} \Sigma^{-1 / 2}$, and $\left\{\boldsymbol{v}_{i}\right\}$ are the $r$ eigenvectors of $\Sigma^{-1 / 2} C(\boldsymbol{h})^{\prime} \Sigma^{-1} C(\boldsymbol{h}) \Sigma^{-1 / 2}$. We require that $\operatorname{cov}\left(W_{i}(\boldsymbol{s}), W_{i^{\prime}}\left(\boldsymbol{s}^{\prime}\right)\right)=0 \quad \forall i \neq$ $i^{\prime}, \forall \boldsymbol{s}, \boldsymbol{s}^{\prime}$. This implies that $\operatorname{cov}\left(\boldsymbol{p}_{i}^{\prime} \boldsymbol{Z}(\boldsymbol{s}+\boldsymbol{h}) \Leftrightarrow \boldsymbol{q}_{i}^{\prime} \boldsymbol{Z}(\boldsymbol{s}), \boldsymbol{p}_{i^{\prime}}^{\prime} \boldsymbol{Z}\left(\boldsymbol{s}^{\prime}+\boldsymbol{h}\right) \Leftrightarrow \boldsymbol{q}_{i^{\prime}}^{\prime} \boldsymbol{Z}\left(\boldsymbol{s}^{\prime}\right)\right)=$ 0 for $s=s^{\prime}$ and $\forall i \neq i^{\prime}$. That is, we require

$$
\boldsymbol{p}_{i}^{\prime} \Sigma \boldsymbol{p}_{i^{\prime}}+\boldsymbol{q}_{i}^{\prime} \Sigma \boldsymbol{q}_{i^{\prime}} \Leftrightarrow \boldsymbol{p}_{i}^{\prime} C(\boldsymbol{h}) \boldsymbol{q}_{i^{\prime}} \Leftrightarrow \boldsymbol{q}_{i}^{\prime} C(\Leftrightarrow \boldsymbol{h}) \boldsymbol{p}_{i^{\prime}}=0, \forall i \neq i^{\prime} .
$$

But this is true because we know that $P \Sigma P=I=Q^{\prime} \Sigma Q$, and $P^{\prime} C(\boldsymbol{h}) Q=$ $T=\operatorname{diag}\left(\tau_{1}, \ldots, \tau_{r}\right)$.

For $\boldsymbol{h}$ fixed, the SVD (equivalently, canonical correlation) analysis yields a set of processes $\left\{\boldsymbol{p}_{i}^{\prime} \boldsymbol{Z}(\boldsymbol{s}+\boldsymbol{h}) \Leftrightarrow \boldsymbol{q}_{i}^{\prime} \boldsymbol{Z}(\boldsymbol{s})\right\}$ that are substitutes for the spatial factors $\left\{W_{i}(\boldsymbol{s})\right\}$. These depend on a choice of $\boldsymbol{h}$ in much the same way that Switzer and Green's (1984) MAFs do. We investigate their stability as $\boldsymbol{h}$ is varied and their power as an exploratory tool for building shifted-lag models.

\subsection{Interactive and Dynamic Graphical Tools}

Two aspects of the methods discussed in the previous sections are ideally approached with interactive and dynamic graphical tools. The first is study- 
ing linear combinations of $\boldsymbol{Z}(\boldsymbol{s}+\boldsymbol{h})$ versus $\boldsymbol{Z}(\boldsymbol{s})$ in a more flexible manner than that allowed by canonical correlation analysis alone, and the second is studying the effect of varying $\boldsymbol{h}$ by providing interactive controls. The work that we describe is new but there is some history to the ideas which we now relate.

In canonical correlation analysis, as discussed in the previous sections, the problem is to predict $\boldsymbol{y}=\left(y_{1}, \ldots, y_{q}\right)^{\prime}$ from $\boldsymbol{x}=\left(x_{1}, \ldots, x_{p}\right)^{\prime}$. When the solution is restricted to linear predictors, similar reasoning to that given in Section 2 shows that the optimal predictor is given by the linear combinations $\boldsymbol{u}=A \boldsymbol{x}$ and $\boldsymbol{v}=B \boldsymbol{y}$ which minimize $\mathrm{E}\left[\|\boldsymbol{v} \Leftrightarrow \boldsymbol{u}\|^{2}\right]$, when assuming $\mathrm{E}[\boldsymbol{v} \Leftrightarrow \boldsymbol{u}]=$ $\mathbf{0}$, and where $A$ and $B$ each have $r$ linearly independent rows satisfying $A \cdot \operatorname{var}(\boldsymbol{x}) \cdot A^{\prime}=I$ and $B \cdot \operatorname{var}(\boldsymbol{y}) \cdot B^{\prime}=I$. This can be solved algebraically (for example, via SVD) but a more general and flexible approach is to visualize the minimization process and to incorporate user interaction.

Consider arbitrary linear combinations of $\boldsymbol{x}$ and $\boldsymbol{y}: \boldsymbol{\alpha}^{\prime} \boldsymbol{x}$ and $\boldsymbol{\beta}^{\prime} \boldsymbol{y}$ where $\boldsymbol{\alpha} \in \mathcal{S}^{p-1}\left(\mathcal{S}^{p-1}\right.$ is a $(p \Leftrightarrow 1)$-sphere in $\left.\mathbb{R}^{p}\right)$ and $\boldsymbol{\beta} \in \mathcal{S}^{q-1}$. The sequence of projections

$$
\mathcal{T}=\left\{\left(\boldsymbol{\alpha}(t)^{\prime} \boldsymbol{x}, \boldsymbol{\beta}(t)^{\prime} \boldsymbol{y}\right): t \text { is time-continuous on } \mathbb{R}\right\}
$$

shown dynamically is defined as a correlation tour (Buja et al., 1988). The term "correlation" derives directly from canonical correlation analysis. The word "tour" makes the analogy to the automatic motion algorithm generating movement over $\mathcal{T}$ developed for the grand tour (Asimov, 1985). The application of a correlation tour has broad scope. For example, in the regression setting $(q=1)$ let the vertical axis display be the residuals after fitting the linear model $y=\boldsymbol{x}^{\prime} \boldsymbol{\theta}+\boldsymbol{\varepsilon}$. The correlation tour plots the residuals against arbitrary linear combinations of $\boldsymbol{x}$ allowing more general checking of the model fit than that given by plotting the residuals versus $x_{1}, \ldots, x_{p}$, and the fitted values. For our purpose, set

$$
\boldsymbol{x}=\boldsymbol{Z}(\boldsymbol{s}) \text { and } \boldsymbol{y}=\boldsymbol{Z}(\boldsymbol{s}+\boldsymbol{h}),
$$

and consider linear combinations of the form

$$
\boldsymbol{\alpha}_{\Sigma}=\Sigma^{-1 / 2} \boldsymbol{\alpha}\left(\in \mathcal{E}^{p-1}\right) \text { and } \boldsymbol{\beta}_{\Sigma}=\Sigma^{-1 / 2} \boldsymbol{\beta}\left(\in \mathcal{E}^{q-1}\right)
$$

where $\mathcal{E}^{p-1}$ is an ellipse in $\mathbb{R}^{p}$ of the form $\left\{\boldsymbol{w} \in \mathbb{R}^{p}: \boldsymbol{w}^{\prime} \Sigma \boldsymbol{w}=1\right\}$ (and similarly for $\mathcal{E}^{q-1}$ ). We are interested in finding the linear combinations of $\boldsymbol{Z}(\boldsymbol{s})$ which best predict $\boldsymbol{Z}(\boldsymbol{s}+\boldsymbol{h})$. The sequence of projections

$$
\mathcal{T}_{\Sigma}=\left\{\left(\boldsymbol{\alpha}_{\Sigma}(t)^{\prime} \boldsymbol{x}, \boldsymbol{\beta}_{\Sigma}(t)^{\prime} \boldsymbol{y}\right): t \text { is time-continuous on } \mathbb{R}\right\}
$$

is shown dynamically. In the implementation, $\Sigma$ will be estimated by the sample variance-covariance matrix of $\left\{\boldsymbol{Z}\left(\boldsymbol{s}_{1}\right), \ldots, \boldsymbol{Z}\left(\boldsymbol{s}_{n}\right)\right\}$.

In addition to allowing the directions of movement, $\boldsymbol{\alpha}, \boldsymbol{\beta}$, to vary randomly (like that provided in the "Data Viewer" (Buja et al., 1988)), we use 

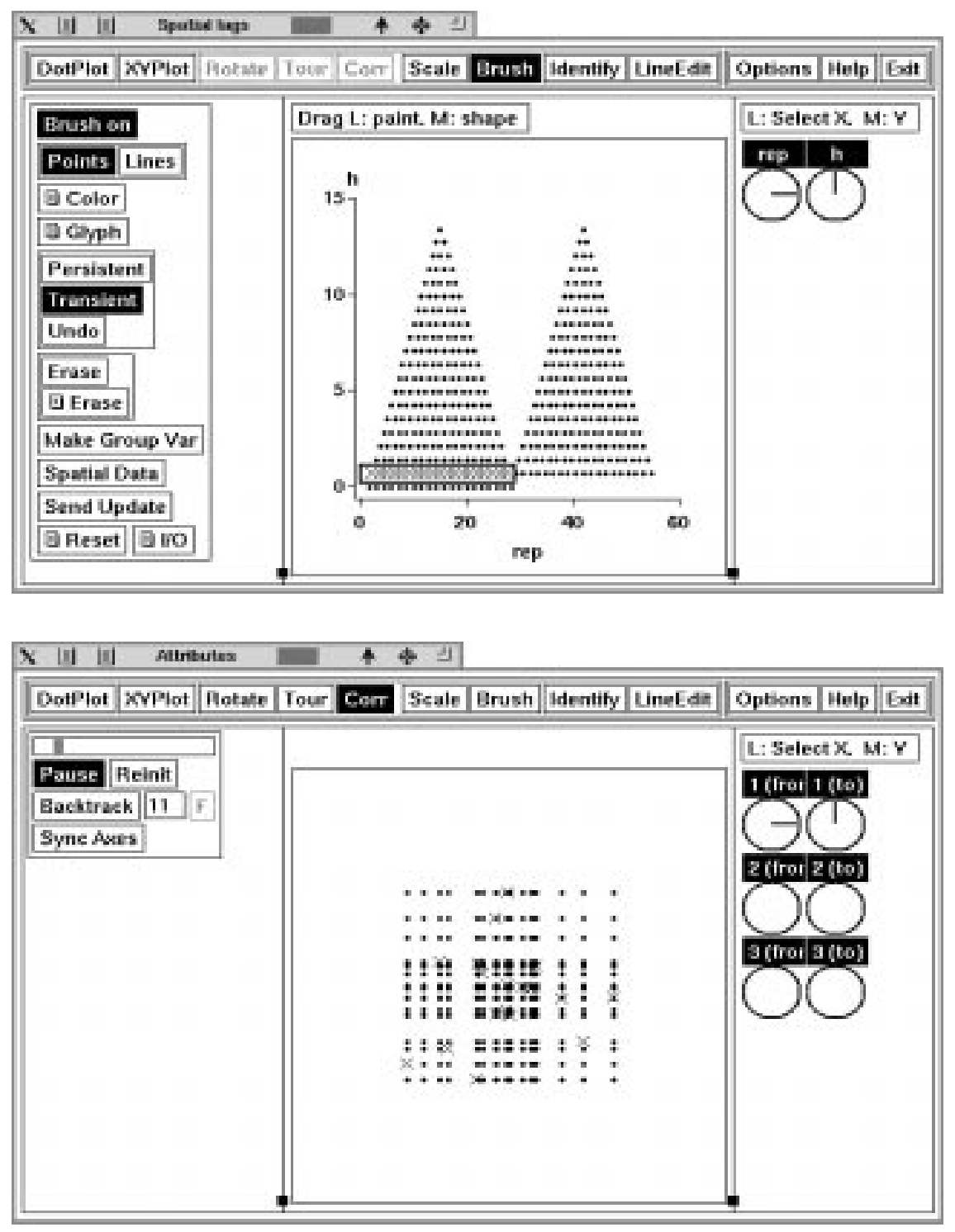

Figure 4: Brushing on spatial lags linked to a correlation tour in XGobi. The top plot shows XGobi in Brush mode with all spatial lags plotted, and lag $h=1$ are brushed $(x)$. The bottom plot shows XGobi in Corr mode, at the starting projection $\left(Z_{1}(\boldsymbol{s}), Z_{1}(\boldsymbol{s}+\boldsymbol{h})\right)$ of a correlation tour on $\boldsymbol{Z}(\boldsymbol{s})$ versus $Z(s+h)$. 
the quantity $\Leftrightarrow \mathrm{E}\left[\left\|\boldsymbol{\beta}_{\Sigma}^{\prime} \boldsymbol{Z}(\boldsymbol{s}+\boldsymbol{h}) \Leftrightarrow \boldsymbol{\alpha}_{\Sigma}^{\prime} \boldsymbol{Z}(\boldsymbol{s})\right\|^{2}\right]$ (or, equivalently, $\operatorname{corr}\left(\boldsymbol{\beta}_{\Sigma}^{\prime} \boldsymbol{Z}(\boldsymbol{s}+\right.$ $\left.\boldsymbol{h}), \boldsymbol{\alpha}_{\Sigma}^{\prime} \boldsymbol{Z}(\boldsymbol{s})\right)$ ) as an index $I_{S C}$ (Index of Spatial Continuity) to direct the movement of the correlation tour in the direction that maximizes $I_{S C}$. In practice, $\Leftrightarrow E\left[\left\|\boldsymbol{\beta}_{\Sigma}^{\prime} \boldsymbol{Z}(\boldsymbol{s}+\boldsymbol{h}) \Leftrightarrow \boldsymbol{\alpha}_{\Sigma}^{\prime} \boldsymbol{Z}(\boldsymbol{s})\right\|^{2}\right]$ is estimated by a sample average. The maximum of $I_{S C}$ is the largest eigenvalue, $\tau_{1}$, and $\left(\boldsymbol{\alpha}_{\Sigma}, \boldsymbol{\beta}_{\Sigma}\right)$ are the associated eigenvectors (called $\boldsymbol{p}_{1}, \boldsymbol{q}_{1}$ in Section 2.1). To be identical to the procedure discussed in Section 2.1 we would proceed iteratively, to tour in the orthogonal subspaces to find the remaining $r \Leftrightarrow 1$ eigenvalues and corresponding eigenvectors. There are several advantages of this graphical approach. We have finessed the need to estimate the covariance matrix $C(\boldsymbol{h})$. We have a visual summary of the lack of symmetry in $C(\boldsymbol{h})$ by comparing results for $\boldsymbol{h}$ and $-\boldsymbol{h}$, as described below. Other indices, for example, med $\left|\boldsymbol{\beta}_{\Sigma}^{\prime} \boldsymbol{Z}(\boldsymbol{s}+\boldsymbol{h}) \Leftrightarrow \boldsymbol{\alpha}_{\Sigma}^{\prime} \boldsymbol{Z}(\boldsymbol{s})\right|^{1 / 2}$, may also be considered. Finally, features of the data, such as outliers, affecting the numerical solutions, can be detected visually and addressed as an integral part of the process. If $\boldsymbol{\alpha}_{\Sigma}, \boldsymbol{\beta}_{\Sigma}$ are synchronized so that $\boldsymbol{\alpha}_{\Sigma} \equiv \boldsymbol{\beta}_{\Sigma}$ then the process is analogous to finding the MAFs suggested by Switzer and Green (1984).

The nature of fixing $\boldsymbol{h}$ is effectively subsetting on pairwise spatial displacement and augmenting the attribute variables with a spatially lagged set of variables, effectively doubling the number of variables. Allowing $\boldsymbol{h}$ to vary enables one to study and compare different subsets. To see this, consider

$$
N(\boldsymbol{h})=\left\{\left(\boldsymbol{s}_{i}, \boldsymbol{s}_{j}\right): \boldsymbol{s}_{i} \Leftrightarrow \boldsymbol{s}_{j}=\boldsymbol{h} ; \boldsymbol{s}_{i}, \boldsymbol{s}_{j} \in D\right\} .
$$

For example, let $\operatorname{dim}(D)=1$; then varying $h$ can be achieved by interactively brushing in a plot of $h$. Figure 4 shows an example on a data set with observations taken at regularly spaced points. All pairwise locations, $\left\{\left(s_{i}, s_{j}\right): s_{i}, s_{j} \in D\right\}$, are plotted and these form a grid in $\mathbb{R}^{2}$; points of zero distance lie along the diagonal $s_{i}=s_{j}$, points with $s_{i}<s_{j}(h>0)$ are in the upper triangle, and points with $s_{i}>s_{j}(h<0)$ are on the lower triangle. This form is awkward to brush using the usual rectangular brush, so we have rotated the points $45^{\circ}$ and split them at $h=0$ so that the triangle of points on the left represent those with $h>0$ and points on the right represent those with $h<0$. This format also allows brushing on $|h|$ to check symmetry of $C(h)$. Figure 4 shows two window dumps, one displays the spatial lags in the manner described above with lag $h=1$ brushed as $\times$ and the second plot shows a snapshot, $\left(Z_{1}(s), Z_{1}(s+h)\right)$, of a correlation tour on $\left(\boldsymbol{Z}(s)^{\prime}, \boldsymbol{Z}(s+h)^{\prime}\right)^{\prime}$ with corresponding points brushed. (The data is simulated from a shiftedlag model containing no covariance structure.) Erase brushing can be used instead of glyph brushing to concentrate attention on specific lags only. The method is also general enough to be used when the observations are made on irregular intervals. When $\operatorname{dim}(D)=2$, it may be of more interest to consider $\boldsymbol{h}$ as an angle, a magnitude, and allow brushing on angle or magnitude or both.

The usefulness of varying $\boldsymbol{h}$ can be appreciated by considering the methods discussed in Cressie and Majure (1993). There, plots of 9 pairwise com- 
binations of 3 attributes are examined at different spatial lags. To reduce the visual overload, summary measures are computed for each pair and lag. These numerical measures are plotted by lag for each pair of variables and these plots are examined to gain insight into spatial dependence over lags. This is a process beautifully suited to an interactive environment, where the user can change pairwise plots at will and brush (subset) on spatial lags interactively.

\section{Integration of Interactive and Dynamic Graphics Tools into a GIS}

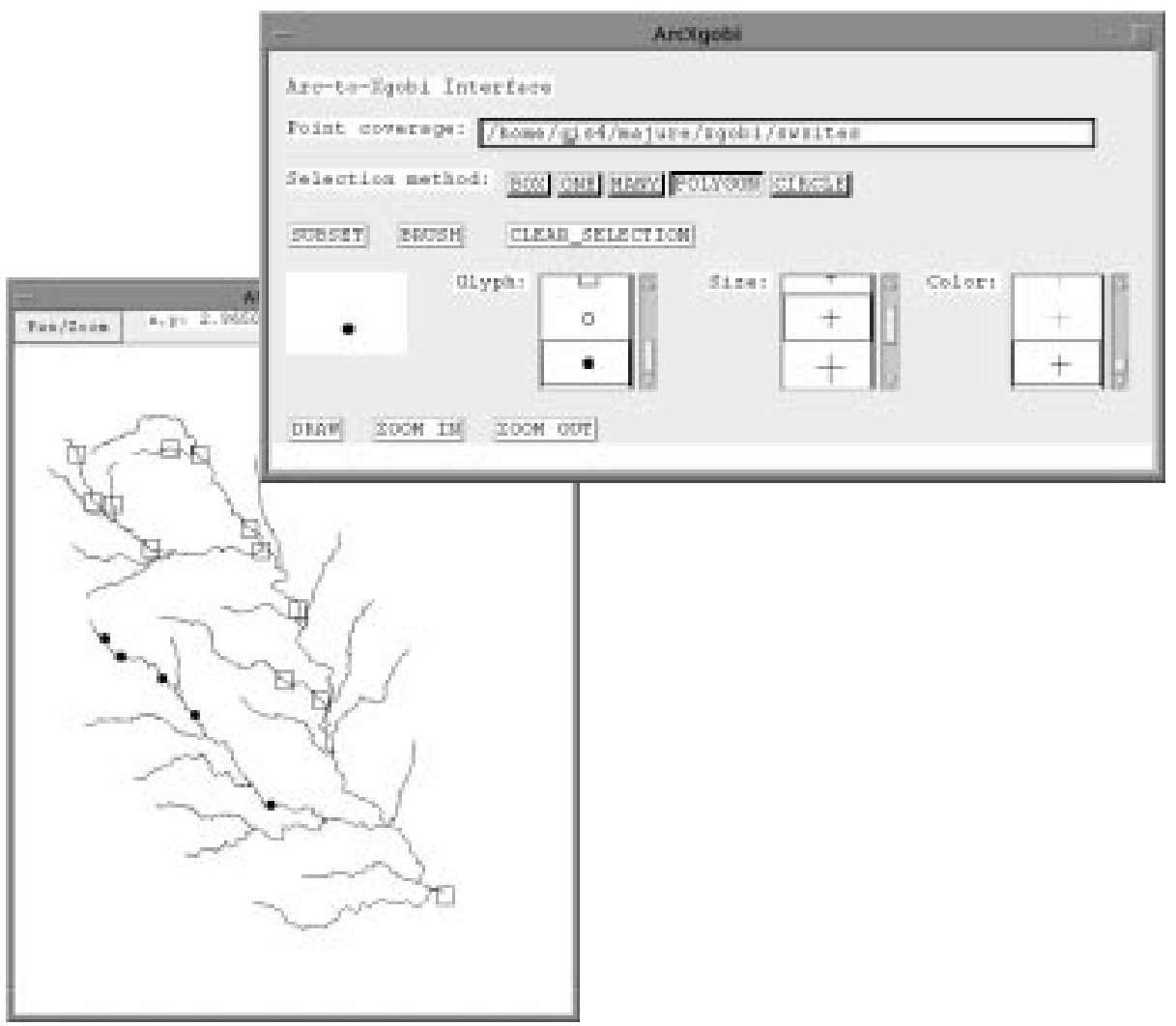

Figure 5: ARC/INFO control panel and example map view used in the link with XGobi.

Emphasis in GIS development has been on the input of data, its management (storage, retrieval) and display of maps, graphs, and tables. GISs have some capability to allow statistical analyses but it is generally limited. A number of recent suggestions have been made (Openshaw, 1991; Anselin and Getis, 
1992; Ding and Fotheringham, 1992; Fotheringham and Rogerson, 1993, for example) to redress this imbalance. Our research addresses the extremely important problem of multivariate exploratory spatial data analysis in a GIS. GIS data structures allow the representation of areal features for the storage of information reported at an aggregated spatial level, such as counties or census tracts, and linear features for the storage of information collected from a stream or a transportation network, for example. The topological data structure of a GIS makes it possible to determine spatial relationships between sampling locations, such as stream sites, that would be difficult to determine otherwise. The display capabilities of a GIS allow the spatial variables to be overlaid on a background of hydrography, transportation, population, land use, or other information relevant to the attributes being considered. For example, in Figure 5 the map view shows sampling sites along streams in Erath county, Texas. Information about the topography or land use near a sample site can give valuable insights into the values of attributes collected at the site.

A GIS is intrinsically multivariate and yet this is ignored by the univariate statistical analyses currently available. By building an interface between a GIS and software for dynamic graphics, we will also provide a good platform for developing new graphical methods (for example, those discussed in Section 2.3 ) by facilitating use and testing on data sets available in the GIS.

Our efforts have focused on interfacing the GIS software, ARC/INFO ${ }^{\mathrm{TM}}$ with XGobi (Swayne et al., 1991). XGobi provides interactive and dynamic graphical tools in the X Window System ${ }^{\text {TM }}$ environment for exploring multivariate data through the manipulation of scatterplots. ARC/INFO is used to maintain the data base and to display the geography, while XGobi primarily is used to explore the relationships amongst the attributes. Figure 6 shows how the communication between these two programs is established.

Before any connection can be established, ARC/INFO and an ARC/XGobi interface process must be activated on the same host where the ARC/INFO data base is maintained. An XGobi process, that is, a client either residing on the same host or anywhere else, that wants to use some ARC/INFO data sets has to connect to the ARC/XGobi interface process, that is, the server. The XGobi client can select whether it wants the data set that is currently selected within ARC/INFO and possible updates of this selection or only static data that is available in the ARC/INFO data base. In the latter case it is not even required that ARC/INFO is running. The interprocess communication is based on Stevens' (1990) concurrent server example, and uses a TCP (Internet stream) socket. This setup implies that upon receiving a connection request from an XGobi client, the ARC/XGobi server forks an identical child process, and thus establishes a one-to-one connection between an ARC/XGobi server and an XGobi client. A more detailed description of

TM $A R C / I N F O$ is a trademark of Environmental Systems Research Institute, Inc.

TM $X$ Window System is a trademark of MIT. 


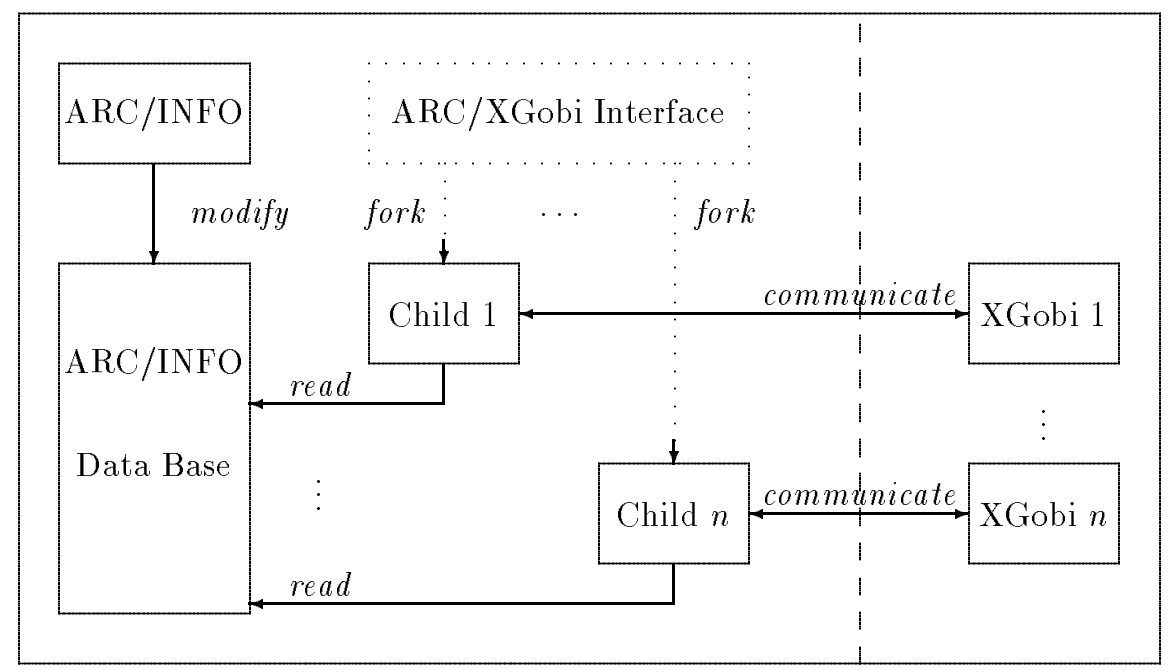

Figure 6: Interface linking ARC/INFO with XGobi.

this link can be found in Symanzik et al. (1994).

The main purpose of the ARC/XGobi servers (children) is to continuously check whether the ARC/INFO data base has been changed. Changes to the ARC/INFO data base reflect the results of brushing and subsetting operations conducted through the ARC/INFO control panel shown in Figure 5 . When changes are detected the newly brushed or subsetted points are immediately passed to each XGobi client. The user can brush the sampling locations in the map view using a variety of brush types and choice of glyphs, with different size and color combinations. The choice has been coordinated to reflect those available in XGobi. The attribute values currently visible in XGobi that are linked to the marked coordinates will instantaneously obtain the same glyph. So, this interface allows the user to link views interactively such that modifications of one view automatically change the other views in the different XGobi clients.

\section{Summary}

In the analysis of spatial data, it is desirable to keep the spatial variables firmly rooted in the geography of the area of interest and also provide a high level of interaction with the data using dynamic graphics tools. With this in mind, we have built a link between the GIS software ARC/INFO and software for interactively exploring multivariate data with dynamic graphics, XGobi. The combination provides tools for multivariate exploratory spatial data analysis for examining both large-scale variation (trend) and small-scale variation (spatial dependence) in the attribute variables, $Z(s)$, and a plat- 
form for exploring new methods. We have focused on developing tools for detecting spatial dependence, in particular, as applied to shifted-lag models. The graphical tools are dynamic involving displays of linear combinations of $\boldsymbol{Z}(\boldsymbol{s}+\boldsymbol{h})$ versus $\boldsymbol{Z}(\boldsymbol{s})$, in a correlation tour. The effectiveness and usefulness of the methods shall be assessed on simulated and actual data.

\section{Acknowledgements}

Cressie's research was supported by the National Science Foundation, the National Security Agency, and the Office of Naval Research. Symanzik was partially supported by a German "DAAD-Doktorandenstipendium aus Mitteln des zweiten Hochschulsonderprogramms".

\section{References}

Akaike, H. (1976). Canonical Correlation Analysis and the Use of an Information Criterion. In Mehra, R. K. and Lainiotis, D. J., editors, Systems Identification: Advances and Case Studies. Academic Press, New York, NY, pages 27-96.

Anderson, T. W. (1958). An Introduction to Multivariate Statistical Analysis. Wiley, New York, NY.

Andrews, D. F. and Herzberg, A. M. (1985). Data - A Collection of Problems from Many Fields for the Student and Research Worker. Springer-Verlag, New York, NY.

Anselin, L. and Getis, A. (1992). Spatial Statistical Analysis and Geographic Information Systems. Annals of Regional Science, 26:19-33.

Aoki, M. (1987). State Space Modelling of Time Series. Springer, New York, NY.

Asimov, D. (1985). The Grand Tour: A Tool for Viewing Multidimensional Data. SIAM Journal Scientific and Statistical Computing, 6(1):128-143.

Box, G. E. P. and Tiao, G. C. (1977). A Canonical Analysis of Multiple Time Series. Biometrika, 64:355-365.

Bradley, R. and Haslett, J. (1992). High-interaction Diagnostics for Geostatistical Models of Spatially Referenced Data. The Statistician, 41:371-380.

Buja, A. and Asimov, D. (1986). Grand Tour Methods: An Outline. Computing Science and Statistics, 17:63-67.

Buja, A., Asimov, D., Hurley, C., and McDonald, J. A. (1988). Elements of a Viewing Pipeline for Data Analysis. In Cleveland, W. S. and McGill, M. E., editors, Dynamic Graphics for Statistics, pages 277-308. Wadsworth, Monterey, CA.

Buja, A., McDonald, J. A., Michalak, J., and Stuetzle, W. (1991). Interactive Data Visualization using Focusing and Linking. In Nielson, G. M. and Rosenblum, L., editors, Proceedings of Visualization '91. IEEE Computer Society Press, Los Alamitos, CA, pages 156-162.

Chauvet, P. (1982). The Variogram Cloud. In Proceedings of the 17th APCOM Symposium. Colorado School of Mines, Golden, CO, pages 757-764.

Cooper, D. M. and Wood, E. F. (1982). Identifying Multivariate Time Series Models. Journal of Time Series Analysis, 3:153-164.

Cressie, N. (1984). Towards Resistant Geostatistics. In Verly, G., David, M., Journel, A., and Marechal, A., editors, Geostatistics for Natural Resources Characterization, Part 1. Reidel, Dordrecht, pages 21-44.

Cressie, N. and Helterbrand, J. D. (1994). Multivariate Spatial Statistical Models. Geographical Systems, 1. Forthcoming. 
Cressie, N. and Majure, J. (1993). Visualizing Spatial Dependence in Multivariate Data. Technical Report 93-4, ISU Statistical Laboratory Preprint Series.

Cressie, N. A. C. (1993). Statistics for Spatial Data (revised edition). Wiley, New York, NY.

Dillon, W. R. and Goldstein, M. (1984). Multivariate Analysis: Methods and Applications. Wiley, New York, NY.

Ding, Y. and Fotheringham, A. S. (1992). The Integration of Spatial Analysis and GIS. Computers, Environment and Urban Systems, 16:3-19.

Fotheringham, A. S. and Rogerson, P. (1993). GIS and Spatial Analytical Problems. International Journal of Geographical Information Systems, 7:3-19.

Getis, A. and Ord, J. K. (1992). The Analysis of Spatial Association by Use of Distance Statisics. Geographical Analysis, 24:189-206.

Goodchild, M. F. (1991). The Technological Setting of GIS. In Maguire, D. J., Goodchild, M. F., and Hind, D. W., editors, Geographical Information Systems: Principles and Applications, vol. 1. Longman, London, pages 9-20.

Goodchild, M. F., Haining, R. P., and Wise, S. (1992). Integrating GIS and Spatial Analysis - problems and possibilities. International Journal of Geographical Information Systems, 6:407-423.

Haslett, J., Bradley, R., Craig, P., Unwin, A., and Wills, G. (1991). Dynamic Graphics for Exploring Spatial Data with Application to Locating Global and Local Anomalies. The American Statistician, 45(3):234-242.

Maguire, D. J. (1991). An Overview and Definition of GIS. In Maguire, D. J., Goodchild, M. F., and Hind, D. W., editors, Geographical Information Systems: Principles and Applications, vol. 1. Longman, London, pages 9-20.

McDonald, J. A. and Willis, S. (1987). Use of the Grand Tour in Remote Sensing. ASA Video Collection, American Statistical Association, Alexandria, VA.

Openshaw, S. (1991). Developing Appropriate Spatial Analysis Methods for GIS. In Maguire, D. J., Goodchild, M. F., and Hind, D. W., editors, Geographical Information Systems: Principles and Applications, vol. 1. Longman, London, pages $389-402$.

Pena, D. and Box, G. E. P. (1987). Identifying a Simplifying Structure in Time Series. Journal of the American Statistical Association, 82:836-843.

Stevens, W. R. (1990). UNIX Network Programming. Prentice-Hall, Englewood Cliffs, NJ.

Swayne, D. F., Cook, D., and Buja, A. (1991). XGobi: Interactive Dynamic Graphics in the $\mathrm{X}$ Window System with a Link to S. In ASA Proceedings of the Section on Statistical Graphics. American Statistical Association, Alexandria, VA, pages $1-8$.

Switzer, P. and Green, A. A. (1984). Min/max Autocorrelation Factors for Multivariate Spatial Imagery. Technical Report 6, Department of Statistics, Stanford University.

Symanzik, J., Majure, J., Cook, D., and Cressie, N. (1994). Dynamic Graphics in a GIS: A Link between ARC/INFO and XGobi. Computing Science and Statistics, 26. To appear.

Tiao, G. C. and Tsay, R. S. (1989). Model Specification in Multivariate Time Series. Journal of the Royal Statistical Society, Series B, 51:157-195.

Wackernagel, H. (1988). Geostatistical Techniques for Interpreting Multivariate Spatial Information. In Chung, C. F., Fabbri, A. G., and Sinding-Larsen, R., editors, Quantative Analysis of Mineral and Energy Resources. Reidel, Dordrecht, pages 393-409. 